\title{
Perfil energético e hormonal de ovelhas Santa Inês do terço médio da gestação ao pós-parto ${ }^{1}$
}

\author{
Carolina A.S.C. Araujo ${ }^{2 *}$, João P. Nikolaus², Aline A. Morgado², Bruno M. Monteiro², \\ Frederico A.M.L. Rodrigues ${ }^{2}$, Thales A.F. Vechiato ${ }^{2}$, Pierre C. Soares ${ }^{3}$ \\ e Maria C.A. Sucupira ${ }^{2}$
}

\begin{abstract}
Araujo C.A.S.C., Nikolaus J.P., Morgado A.A., Monteiro B.M., Rodrigues F.A.M.L., Soares P.C. \& Sucupira M.C.A. 2014. [Energetic and hormonal profile of Santa Ines ewes in the middle of gestation to postpartum.] Perfil energético e hormonal de ovelhas Santa Inês do terço médio da gestação ao pós-parto. Pesquisa Veterinária Brasileira 34(12):12511257. Departamento de Clínica Médica Veterinária, Faculdade de Medicina Veterinária e Zootecnia, Universidade de São Paulo, Av. Prof. Dr. Orlando Marques de Paiva 87, Cidade Universitária, São Paulo, SP 05508-270, Brazil. E-mail: carolcbpr@gmail.com

Important physiological adaptations occur in the periparturient period; their failure predispose the ewe to metabolic diseases. Knowledge of normal changes makes an early recognition and treatment of mal functions possible and enables prevention of diseases and losses. The biochemical profile of Santa Ines ewes from the $88^{\text {th }}$ day of gestation until 28 days after parturition was evaluated and compared to non pregnant controls. The ewes were divided in groups according to the number of fetuses: G0, non pregnant (10); G1, one (10); G2, two and three fetuses (14). All animals had their heart and respiratory rates as well as their ruminal motility recorded. Serum and plasma was analyzed for the following parameters: glucose, non esterified fatty acids (NEFA), beta hydroxibutyrate (BHB), T3, T4, insulin, glucagon and cortisol activities. Results showed changes in biochemical variables of energy and protein profile during pregnancy and parturition. During the last third of gestation, all ewes showed slightly increased NEFA, T3 and T4 levels when compared to non pregnant ewes. At lambing pregnant ewes, had higher glucose, NEFA and T3 levels. No significant differences on measured parameters comparing simple and multiple gestations were observed. Therefore, when there is adequate adaptation in this period of high metabolic challenge, biochemical parameters considered here are independent of the number of fetuses gestate and can be considered as reference values for a pregnant ewes from the middle third of gestation to first month postnatal period.
\end{abstract}

INDEX TERMS: Energy status, protein status, biochemical profile, hormonal profile, sheep.

RESUMO.- No período periparto ocorrem importantes adequações fisiológicas que, se não forem efetivas predispõem a fêmea a enfermidades metabólicas. 0 conhecimento desta adaptação é relevante para que sejam implementadas, precocemente, medidas preventivas a poupar perdas pro-

\footnotetext{
${ }^{1}$ Recebido em 7 de dezembro de 2013.

Aceito para publicação em 16 de agosto de 2014.

${ }^{2}$ Departamento de Clínica Médica Veterinária (VCM), Faculdade de Medicina Veterinária e Zootecnia (FMVZ), Universidade de São Paulo (USP), Av. Prof. Dr. Orlando Marques de Paiva 87, Cidade Universitária, São Paulo, SP 05508-270, Brasil. *Autor para correspondência: carolcbpr@gmail.com

${ }^{3}$ Departamento de Clínica Médica Veterinária, Faculdade de Medicina Veterinária, Universidade Federal Rural do Pernambuco (UFRPE), Rua Dom Manuel de Medeiros s/n, Bairro Dois Irmãos, Recife, PE 52171-030, Brasil.
}

dutivas. Com este objetivo foi avaliado o perfil energético e hormonal de ovelhas Santa Inês durante a gestação e puerpério. Foram utilizadas 10 ovelhas não gestantes (G0), 10 gestantes de um (G1) e 14 gestantes de dois e três fetos (G2). Foram avaliadas concentrações plasmáticas de glicose, ácidos graxos não esterificados (AGNE), betahidroxibutirato (BHB), e as concentrações séricas de insulina, glucagon, cortisol, triiodotironina $\left(\mathrm{T}_{3}\right)$ e tiroxina $\left(\mathrm{T}_{4}\right)$ a partir do $88^{\circ}$ dia de gestação até o $28^{\circ}$ dia pós-parto. No terço final de gestação, ovelhas gestantes apresentaram maiores concentrações de AGNE, $\mathrm{T}_{3}$ e T4 que as ovelhas não gestantes. No momento do parto foram observadas maiores concentrações de glicose, AGNE e T3 para todas as ovelhas gestantes em relação às não gestantes. Não houve diferença entre 
as ovelhas gestantes de um, dois ou três fetos. As diferenças observadas ocorreram apenas entre ovelhas gestantes e as vazias. Portanto, quando há adequada adaptação neste período de elevado desafio metabólico, os parâmetros bioquímicos aqui considerados independem do número de fetos gestados e podem ser considerados como valores de referência para ovelhas gestantes de um feto ou mais fetos do terço médio de gestação ao primeiro mês pós-parto.

TERMOS DE INDEXAÇÃO: Status energético, status protéico, perfil bioquímico, perfil hormonal, ovinos.

\section{INTRODUÇÃo}

A gestação é importante etapa na fase produtiva da ovelha. Neste estágio as adaptações metabólicas, fisiológicas e anatômicas ocorrem para garantir a formação e o desenvolvimento fetal além da manutenção da vida da mãe e do futuro produto (Reece 2007). Embora ocorram adequações metabólicas durante todo o período gestacional, estas são mais intensas no seu terço final, fase crítica, devido ao maior crescimento dos fetos e ao desenvolvimento da glândula mamária, com consequente aumento das exigências nutricionais (Celi et al. 2008). Qualquer problema que leve ao desequilíbrio entre demanda e aporte de nutrientes para a fêmea nesta fase pode comprometer a saúde e/ou a produção deste animal (Caldeira et al. 2007).

0 perfil energético e hormonal pode ser usado como ferramenta de monitoramento tanto das adaptações-como dos transtornos metabólicos e do desbalanço nutricional (Payne \& Payne, 1987). Fatores como a idade, raça, sexo e estado fisiológico (Reece 2007, Berne \& Levi 2004) podem influenciar na concentração dos constituintes bioquímicos. Acredita-se que haja uma ou um conjunto de variáveis que demonstre precocemente algum desequilíbrio capaz de desencadear transtorno metabólico relacionado ao terço final de gestação, sendo mais frequentes em animais gestando mais de um feto,portanto este estudo teve por objetivo avaliar o perfil energético e hormonal de ovelhas da raça Santa Inês não gestantes, gestantes de um e dois ou três fetos, a fim de verificar se, em situações normais há diferenças marcantes entre estas condições fisiológicas.

\section{MATERIAL E MÉTODOS}

O presente estudo obteve parecer favorável da Comissão de Ética no Uso de Animais (CEUA) da Faculdade de Medicina Veterinária e Zootecnia da Universidade de São Paulo, sob protocolo no 1362 / 2008, aprovado em 23 de abril de 2008.

0 experimento foi conduzido em Piracicaba, nas dependências da Escola Superior de Agricultura Luiz de Queiroz da Universidade de São Paulo (ESALQ-USP). A partir de um lote de 130 ovelhas da raça Santa Inês, foram selecionadas, por meio do exame ultrassonográfico, 41 ovelhas adultas no $60^{\circ}$ dia de gestação. Os animais tinham entre três e sete anos de idade, com escore de condição corporal médio de 3,5 (Russel 1984) e peso corporal médio de $67 \mathrm{~kg}$.

Sete ovelhas foram descartadas do estudo porque apresentaram algum comprometimento associado a sua prole como distocia e toxemia da prenhez. Portanto foram utilizadas 34 ovelhas as quais foram distribuídas em três grupos: dez ovelhas vazias (G0); dez gestantes de um feto (G1) e 14 gestantes de dois ou três fetos (G2).

0 período experimental foi de 98 dias, com início no $88^{\circ}$ dia de gestação e término no $28^{\circ}$ dia pós-parto. A cada 14 dias foram coletadas amostras de sangue e urina, perfazendo oito momentos isto é, M1 = -67; M2 = -3; M3 = -39; M4 = -25; M5 = -11 dias do parto; M6 = momento do parto (até seis horas deste); M7 e M8 aos 14 e 28 dias pós-parto, respectivamente.

Durante todo o estudo as ovelhas receberam ad libitum, dieta total constituída por $20 \%$ de volumoso (bagaço de cana) e $80 \%$ de concentrado (milho, farelo de soja, soja grão e suplemento mineral), que proporcionou 1,77 Mcal/kg de energia liquida (EL), durante todo o período experimental (Quadro 1). Os animais tiveram livre acesso à água.

As amostras de sangue foram obtidas em tubos sem anticoagulante, para a obtenção do soro e tubos contendo fluoreto de sódio, para a obtenção de plasma. Estes foram centrifugados a $500 \mathrm{G}$ por 15 minutos e as alíquotas armazenadas a $-20^{\circ} \mathrm{C}$. No plasma foram analisadas as concentrações de glicose, de ácidos graxos não esterificados (AGNE) e betahidroxibutirato (BHB) e no soro foram determinadas as concentrações dos hormônios insulina, glucagon, cortisol, $\mathrm{T}_{3}$ e $\mathrm{T}_{4}$ Amostras de urina foram obtidas e prontamente analisadas quanto à presença de corpos cetônicos, proteína e glicose por meio do teste de tiras reativas para urina (Combur test ${ }^{\circledR}$ ).

As determinações bioquímicas e hormonais foram realizadas, respectivamente, em analisadores automáticos Lyasis (AMS) no Laboratório de Bioquímica do Departamento de Clínica Médica da Faculdade de Medicina Veterinária da Universidade de São Paulo (FMVZ-USP) e ELECSYS COBAS (Siemens ${ }^{\circledR}$ ) realizado em laboratório particular, utilizando-se procedimentos de análise recomendados por Kaneko et al. (2008).

A estatística descritiva dos dados, representada pelas médias aritméticas e pelos coeficientes de variação (CV) de cada tratamento, foi obtida pelo programa SAS versão 9.2 (SAS/STAT, SAS Institute Inc., Cary, NC). Os testes de normalidade dos resíduos e homogeneidade das variâncias de cada tempo foram realizados utilizando o Guide Data Analise.

As variáveis bioquímicas e hormonais foram analisadas como medidas repetidas no tempo, referentes aos momentos de coleta dos dados (tempo) de acordo com cada tratamento (número de fetos). Quando a premissa de esfericidade não foi respeitada $(\mathrm{P}<0,05)$, as probabilidades de tempo ( $\mathrm{P}$ tempo) e das interações dos tratamentos com o tempo ( $\mathrm{P}$ trat*tempo) foram corrigidas pelo teste de Greenhouse-Geisse Epsilon, utilizando-se o comando Repeated gerado pelo procedimento GLM.

A comparação entre as médias dos grupos dentro de cada tempo (P) foi realizada por meio do teste de médias Least Square Means (LSMeans). Foi utilizado o nível de significância de 5\% para todos os testes realizados. Para a tabulação dos dados e confecção dos gráficos foi utilizado o programa Microsoft Windows Excel, versão 2007.

\section{Quadro 1. Composição da dieta total dos animais durante} período experimental. Piracicaba, São Paulo, 2010

\begin{tabular}{lcc}
\hline Componentes & $\begin{array}{c}\text { Materia seca } \\
\text { (MS) (\%) }\end{array}$ & $\begin{array}{c}\text { Energia líquida } \\
\text { (EL) (Mcal/ kg) }\end{array}$ \\
\hline Milho & 54,3 & 11,1 \\
Farelo de soja (47\% PB) & 9,2 & 1,6 \\
Soja (grão) & 13,8 & 3,4 \\
Calcário & 1,2 & 0,0 \\
Sal mineral* & 1,6 & 0,0 \\
Bagaço de cana & 20,0 & 1,6
\end{tabular}

* Sal mineral: Ovinophós ${ }^{\circledR}$ Tortuga. 


\section{RESULTADOS}

A glicemia aumentou no momento do parto para os grupos de ovelhas prenhes independentemente do número de fetos (Quadro 2, Fig.1A). Foi observada também maior concentração de AGNE no terço final de gestação no parto para as ovelhas gestantes em relação às vazias (Quadro 3, Fig.1C). Não foi observada diferença para BHB entre os grupos nos momentos (Fig.1B).

No perfil hormonal (Fig.1D e 1E) não foram detectadas diferenças nas concentrações de insulina e glucagon entre as fêmeas não gestantes e gestantes. Menores médias do cortisol foram registradas 28 dias pós-parto para as ovelhas que gestaram (Quadro 6, Fig 1F). Maiores concentrações de T3 foram observadas no parto e nos momentos imediata-

Quadro 2. Concentração sérica de glicose ( $\mathrm{mmol} / \mathrm{L})$ e respectivos erros padrões da média de ovelhas da raça Santa Inês vazias ( 0 fetos), com gestação simples ( 1 feto) ou gestação gemelar ( 2 ou 3 fetos) durante a gestação, parto e puerpério. Piracicaba. São Paulo, 2010

\begin{tabular}{ccccc}
\hline Momentos $^{1}$ & \multicolumn{3}{c}{ Tratamento, fetos } & \multirow{2}{*}{$\mathrm{P}^{2}$} \\
\cline { 2 - 4 } & 0 & \multicolumn{3}{c}{1} \\
\hline M1 & $3,86^{\mathrm{a}}(0,15)$ & $3,50^{\mathrm{a}}(0,22)$ & $3,05^{\mathrm{b}}(0,11)$ & 0,001 \\
M2 & $3,97(0,37)$ & $3,78(0,20)$ & $3,79(0,19)$ & 0,953 \\
M3 & $3,85(0,17)$ & $3,93(0,21)$ & $4,03(0,29)$ & 0,940 \\
M4 & $3,73(0,14)$ & $3,71(0,23)$ & $3,52(0,15)$ & 0,578 \\
M5 & $3,67(0,12)$ & $3,89(0,23)$ & $3,66(0,11)$ & 0,513 \\
M6 & $3,11^{\mathrm{b}}(0,15)$ & $6,86^{\mathrm{a}}(1,33)$ & $6,17^{\mathrm{a}}(0,90)$ & 0,001 \\
M7 & $3,68(009)$ & $3,60(0,17)$ & $3,71(0,16)$ & 0,863 \\
M8 & $3,25(0,14)$ & $3,34(0,12)$ & $3,37(0,12)$ & 0,798 \\
Média $^{3}$ & 3,64 & 4,08 & 3,91 &
\end{tabular}

As probabilidades para os efeitos de tratamento, tempo e interação tratamento*tempo observadas na análise de medidas repetidas no tempo foram: Tratamento $=0.929$, Tempo $=0.001$, Trat $*$ Tempo $=0.873$. (1) Dias de gestação das ovelhas prenhez de 1 ou 2 e 3 fetos: M1 = 66, M2 = $52, \mathrm{M} 3=38, \mathrm{M} 4=24, \mathrm{M} 5=10$ dias anteriores ao parto, $\mathrm{M} 6=$ parto, $\mathrm{M} 7 \mathrm{e}$ M8 aos 14 e 28 dias pós-parto; (2) Probabilidade de efeito de tratamento dentro de cada tempo, calculada pelo Least Square Means; (3) Média de cada tratamento em todos os tempos.

Quadro 3. Concentração sérica de AGNE ( $\mu \mathrm{mol} / \mathrm{L})$ e respectivos erros padrões da médiade ovelhas da raça Santa Inês vazias ( 0 fetos), com gestação simples ( 1 feto) ou gestação gemelar ( 2 ou 3 fetos) durante a gestação, parto e puerpério. Piracicaba. São Paulo, 2010

\begin{tabular}{ccccc}
\hline Momentos $^{1}$ & \multicolumn{3}{c}{ Tratamento, fetos } & \multirow{2}{*}{ P $^{2}$} \\
\cline { 2 - 4 } & 0 & 1 & 2 ou 32 & \\
\hline M1 & $304,88(39,41)$ & $229,33(20,20)$ & $302,64(32,88)$ & 0,215 \\
M2 & $169,22(23,34)$ & $195,68(31,94)$ & $230,44(34,10)$ & 0,524 \\
M3 & $155,82(35,77)$ & $258,65(39,26)$ & $275,96(47,20)$ & 0,067 \\
M4 & $177,97^{\mathrm{b}}(24,84)$ & $333,46^{\mathrm{a}}(65,96)$ & $304,61^{\mathrm{a}}(33,49)$ & 0,020 \\
M5 & $189,54^{\mathrm{b}}(34,95)$ & $426,30^{\mathrm{a}}(74,04)$ & $376,80^{\mathrm{a}}(49,42)$ & 0,005 \\
M6 & $68,05^{\mathrm{b}}(4,25)$ & $383,20^{\mathrm{a}}(85,71)$ & $421,53^{\mathrm{a}}(61,44)$ & 0,001 \\
M7 & $102,21(6,94)$ & $200,75(41,08)$ & $168,26(27,79)$ & 0,093 \\
M8 & $180,13(29,89)$ & $132,98(17,83)$ & $125,48(21,09)$ & 0,191 \\
Média $^{3}$ & 168,48 & 270,04 & 275,72 &
\end{tabular}

As probabilidades para os efeitos de tratamento, tempo e interação tratamento*tempo observadas na análise de medidas repetidas no tempo foram: Tratamento $=0.552$, Tempo $=0.001$, Trat $*$ Tempo $=0.545$. (1) Dias de gestação das ovelhas prenhez de 1 ou 2 e 3 fetos: M1 =: 66, M2 $=52, \mathrm{M} 3=38, \mathrm{M} 4=24, \mathrm{M} 5=10$ dias anteriores ao parto; $\mathrm{M} 6=$ parto, M7 e M8 aos 14 e 28 dias pós-parto; (2) Probabilidade de efeito de tratamento dentro de cada tempo, calculada pelo Least Square Means; (3) Média de cada tratamento em todos os tempos.
Quadro 4. Concentração sérica de T3 (nmol/L) e respectivos erros padrões da médiade ovelhas da raça Santa Inês vazias (0 fetos), com gestação simples (1 feto) ou gestação gemelar ( 2 ou 3 fetos) durante a gestação, parto e puerpério. Piracicaba. São Paulo, 2010

\begin{tabular}{|c|c|c|c|c|}
\hline \multirow[t]{2}{*}{ Momentos $^{1}$} & \multicolumn{3}{|c|}{ Tratamento, fetos } & \multirow[t]{2}{*}{$\mathrm{P}^{2}$} \\
\hline & 0 & 1 & 2 ou 32 & \\
\hline M1 & $3,50(0,27)$ & $3,43(0,19)$ & $3,32(0,28)$ & 0,738 \\
\hline M2 & $4,13(0,68)$ & $3,86(0,18)$ & $4,12(0,34)$ & 0,951 \\
\hline M3 & $3,70(0,31)$ & $4,16(0,28)$ & $4,64(0,52)$ & 0,329 \\
\hline M4 & $3,58(0,40)$ & $4,32(0,38)$ & $4,43(0,30)$ & 0,119 \\
\hline M5 & $4,24^{\mathrm{b}}(0,39)$ & $6,23^{\mathrm{a}}(0,46)$ & $5,98^{\mathrm{a}}(0,44)$ & 0,009 \\
\hline M6 & $3,42^{\mathrm{b}}(0,23)$ & $6,34^{\mathrm{a}}(0,68)$ & $6,26^{\mathrm{a}}(0,49)$ & 0,001 \\
\hline M7 & $3,84^{\mathrm{b}}(0,34)$ & $5,86^{\mathrm{a}}(0,46)$ & $5,63^{\mathrm{a}}(0,65)$ & 0,017 \\
\hline M8 & $3,85(0,41)$ & $4,75(0,22)$ & $4,75(0,40)$ & 0,080 \\
\hline Média $^{3}$ & 3,78 & 4,87 & 4,89 & \\
\hline
\end{tabular}

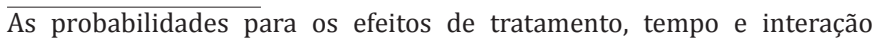
tratamento*tempo observadas na análise de medidas repetidas no tempo foram: Tratamento $=0.011$, Tempo $=0.001$, Trat $*$ Tempo $=0.015$. (1) Dias de gestação das ovelhas prenhez de 1 ou 2 e 3 fetos: $\mathrm{M} 1=66, \mathrm{M} 2=$ 52, M3 = 38, M4 = 24, M5 = 10 dias anteriores ao parto; M6 = parto, M7 e M8 aos 14 e 28 dias pós-parto; (2) Probabilidade de efeito de tratamento dentro de cada tempo, calculada pelo Least Square Means; (3) Média de cada tratamento em todos os tempos.

Quadro 5. Concentração sérica de T4 (nmol/L) e respectivos erros padrões da média de ovelhas da raça Santa Inês vazias (0 fetos), com gestação simples (1 feto) ou gestação gemelar ( 2 ou 3 fetos) durante a gestação, parto e puerpério. Piracicaba. São Paulo, 2010

\begin{tabular}{ccccc}
\hline Momentos $^{1}$ & \multicolumn{3}{c}{ Tratamento, fetos } & \multirow{2}{*}{ P $^{2}$} \\
\cline { 2 - 4 } & 0 & 1 & 2 ou 3 & \\
\hline M1 & $188,82(10,21)$ & $175,51(7,07)$ & $184,03(14,99)$ & 0,789 \\
M2 & $170,79(10,52)$ & $205,12(14,95)$ & $214,31(13,22)$ & 0,065 \\
M3 & $154,89(7,45)$ & $186,61(9,64)$ & $191,81(19,03)$ & 0,247 \\
M4 & $181,39(8,16)$ & $204,39(13,74)$ & $223,01(14,19)$ & 0,088 \\
M5 & $207,98^{\mathrm{b}}(7,61)$ & $270,72^{\mathrm{a}}(14,05)$ & $254,51^{\mathrm{a}}(14,87)$ & 0,011 \\
M6 & $203,31(4,90)$ & $221,93(14,14)$ & $206,56(12,46)$ & 0,568 \\
M7 & $206,23(8,54)$ & $255,97(15,99)$ & $229,28(14,79)$ & 0,078 \\
M8 & $180,59(7,96)$ & $220,65(12,96)$ & $212,62(14,52)$ & 0,106 \\
Média $^{3}$ & 186,75 & 217,61 & 214,51 &
\end{tabular}

As probabilidades para os efeitos de tratamento, tempo e interação tratamento*tempo observadas na análise de medidas repetidas no tempo foram: Tratamento $=0.101$, Tempo $=0.001$, Trat $*$ Tempo $=0.026 .(1)$ Dias de gestação das ovelhas prenhez de 1 ou 2 e 3 fetos: $M 1=66, M 2=$ 52, M3 = 38, M4 = 24, M5 = 10 dias anteriores ao parto; M6 = parto, M7 e M8 aos 14 e 28 dias pós-parto; (2) Probabilidade de efeito de tratamento dentro de cada tempo, calculada pelo Least Square Means; (3) Média de cada tratamento em todos os tempos.

mente antes e após o mesmo para as fêmeas gestantes em relação às não gestantes (Quadro 4, Fig.1G) e maiores valores de T4 foram registrados para as fêmeas gestantes no momento anterior ao parto (Quadro 5, Fig.1H).

Durante todo o período de avaliação não foram observados corpos cetônicos, proteínas e glicose na urina.

\section{DISCUSSÃO}

Sabe-se que o periparto é um período crítico, e que nele há maior possibilidade de ocorrerem perdas econômicas (Caldeira et al. 2007). Estas perdas normalmente decorrentes de manejo inadequado podem desencadear problemas metabólicos, nutricionais, reprodutivos dentre outros (Caldeira et al. 2007). Neste contexto, a determinação do perfil 
Quadro 6. Concentração sérica de cortisol (nmol/L) e erros padrões da média de ovelhas da raça Santa Inês vazias (0 fetos), com gestação simples (1 feto) ou gestação gemelar ( 2 ou 3 fetos) durante a gestação, parto e puerpério. Piracicaba. São Paulo, 2010

\begin{tabular}{ccccc}
\hline Momentos $^{1}$ & \multicolumn{3}{c}{ Tratamento, fetos } & \multirow{2}{*}{$\mathrm{P}^{2}$} \\
\cline { 2 - 4 } & 0 & \multicolumn{3}{c}{1} \\
\hline M1 & $96,70(27,8)$ & $69,22(15,8)$ & $71,41(11,2)$ & 0,803 \\
M2 & $125,78(25,5)$ & $150,45(35,3)$ & $110,79(20,4)$ & 0,818 \\
M3 & $97,04(18,2)$ & $88,31(25,5)$ & $94,32(13,4)$ & 0,844 \\
M4 & $154,04(25,0)$ & $128,90(22,4)$ & $113,46(26,0)$ & 0,268 \\
M5 & $157,87(18,5)$ & $119,76(23,6)$ & $89,16(18,1)$ & 0,062 \\
M6 & $70,12(9,00)$ & $91,73(22,4)$ & $155,04(41,1)$ & 0,112 \\
M7 & $121,56(14,2)$ & $76,90(16,1)$ & $78,24(13,1)$ & 0,070 \\
M8 & $134,13^{\mathrm{a}}(25,05)$ & $75,32^{\mathrm{b}}(21,2)$ & $60,71^{\mathrm{b}}(8,9)$ & 0,020 \\
Média $^{3}$ & 119,66 & 103,61 & 101,77 &
\end{tabular}

As probabilidades para os efeitos de tratamento, tempo e interação tratamento*tempo observadas na análise de medidas repetidas no tempo foram: Tratamento $=0.127$, Tempo $<0.001$, Trat $*$ Tempo $=0.135$. (1) Dias de gestação das ovelhas prenhez de 1 ou 2 e 3 fetos: M1 = 66, M2 = 52, M3 = 38, M4 = 24, M5 = 10 dias anteriores ao parto; M6 = parto, M7 e M8 aos 14 e 28 dias pós-parto; (2) Probabilidade de efeito de tratamento dentro de cada tempo, calculada pelo Least Square Means; (3) Média de cada tratamento em todos os tempos.

energético de um rebanho de ovelhas durante a gestação torna-se importante ferramenta para monitorar o status nutricional e detectar possíveis alterações metabólicas decorrentes das modificações no manejo destes animais (Payne \& Payne 1987).

Neste estudo, foi oferecida dieta com alta densidade energética para as ovelhas. A dieta foi formulada segundo recomendações de Susin (1996), para fornecer $20 \%$ a mais de energia líquida para esta categoria animal, resultando em densidade energética de $1,77 \mathrm{Mcal} / \mathrm{kg} / \mathrm{MS}$. De acordo com revisão de Fthenakis et al (2012), não só a administração de baixos teores energéticos na dieta poderiam ocasionar distúrbio metabólico em ovelhas no terço final de gestação como também, a super nutrição energética neste período poderia desencadear o mesmo efeito e ainda causar distocias.

Glicose, BHB e AGNE são metabólitos importantes no monitoramento do status energético em um rebanho, especialmente durante fase da gestação. Os AGNEs embora sejam de grande relevância para avaliar a mobilização de reservas energéticas, é influenciado inclusive por qualquer forma de estresse, o que justifica a elevada amplitude do coeficiente de variação deste experimento. Hipertrigliceridemia e hipercetonemia podem ocorrer nos casos de privação alimentar, volumoso de baixa qualidade e deficiência energética da alimentação. Sabe-se que o organismo compensa déficits energéticos com a mobilização de reservas corporais e, caso o fígado não consiga metabolizar todo ácido graxo proveniente da lipólise há, formação de corpos cetônicos (Ortolani 1994, Rook 2000). De acordo com Souto et al. (2013), o aumento das concentrações de BHB e AGNE no terço final de gestação em ovinos sugere consumo das reservas corporais e teores mais altos desses metabólitos, indicando balanço energético negativo (BEN) (acima de 0,7 mmol/L e 1,91 mmol/L respectivamente).
No presente estudo não foram detectadas diferenças entre os grupos para as concentrações de BHB, no entanto, observou-se elevação nos teores de AGNE no terço final de gestação e no momento do parto de ovelhas gestantes tanto de um como de dois ou três fetos. Isso indica que as ovelhas gestantes, independentemente do número de fetos, mobilizaram reservas corporais e entraram em balanço energético negativo. Foi encontrada correlação positiva moderada entre AGNEs e BHB $(\mathrm{P}=0,001$ e $\mathrm{r}=0,456)$ mostrando que a mobilização de gordura corporal levou ao aumento da concentração de BHB em grau moderado, pois o balanço energético negativo não foi intenso o que provavelmente permitiu maior proporção de oxidação completa do AGNE mobilizado em relação à oxidação parcial no ciclo da cetogênese (Emery et al 1992).

A pesquisa de corpos cetônicos, proteínas e glicose na urina, apresentou resultados negativos em todas as ovelhas em todos os momentos analisados. Ovelhas hígidas, com escore de condição corporal favorável, ou seja, acima de 3,0 e bem nutridas, como as deste estudo, dificilmente apresentariam positividade nas variáveis urinárias analisadas, pois compensaram bem o balanço energético negativo imposto neste período de transição (Contreras et al 1990, Santos et al 2012,).

0 incremento da concentração dos hormônios tireoideanos (HT) nas ovelhas gestantes está relacionado ao seu importante papel no metabolismo energético (Reece 2007). Em animais não gestantes estes hormônios são considerados indicadores do status metabólico e nutricional do rebanho, quando a privação de energia causa decréscimo nos teores de T3 e o excesso de energia tem efeito oposto (Lima 2005, Todini 2007). Nas ovelhas gestantes a maior atividade tireoidiana em relação às não gestantes ocorre devido aos aumentos tanto nas concentrações de proteínas ligadoras, como na secreção de fatores tireotróficos pela placenta e na resposta por parte da pituitária do hormônio tireoestimulante (TSH) que induz a liberação do hormônio tirotrófico (TRH) pelo hipotálamo (Celi et al. 2008).

Os valores aumentados dos dois grupos de gestantes, das concentrações de T4 no M5 e de T3 no periparto, (M5 ao M7) indicam esse incremento na atividade e concordam com as observações feitas por Todini et al. (2007), que observaram aumento nas concentrações de T3 e T4 em cabras no final de gestação. Porém esses resultados contradizem os relatados por Yildiz et al. (2005), que observaram que as concentrações de $\mathrm{T} 4$ diminuíam progressivamente no decorrer da gestação até momento do parto e atribuíram esse decréscimo ao balanço energético negativo (BEN) que a fêmeas entravam no final da gestação. Estes autores, também observaram diferenças nas concentrações dos HT em fêmeas gestantes de dois fetos, com teores de T3 e T4 menores que as gestantes de fetos únicos, especialmente no final da gestação por causa do maior BEN em que se encontravam as primeiras. $\mathrm{O}$ comportamento dos HT no presente estudo também demonstrou que a dieta oferecida foi satisfatória em suprir boa parte da demanda energética das ovelhas gestantes, independentemente do número de fetos, minimizando o BEN. 
(A)

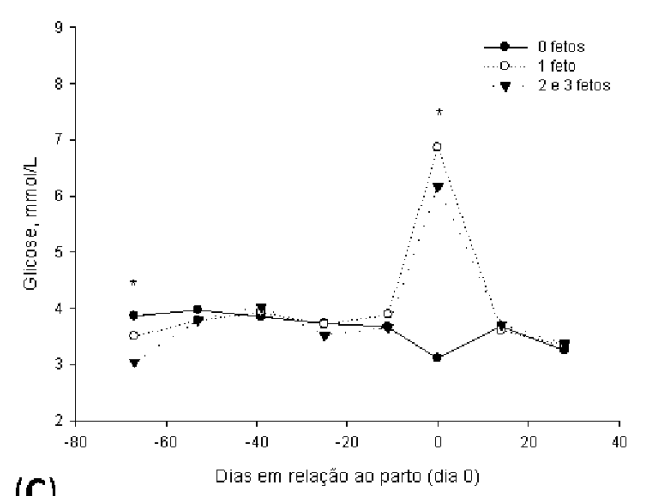

(C)

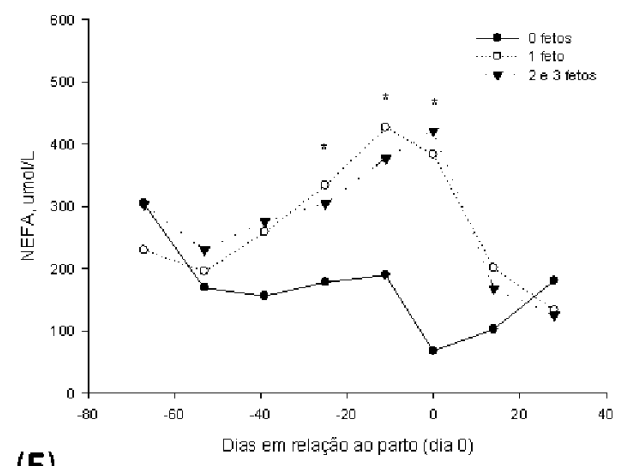

(E)

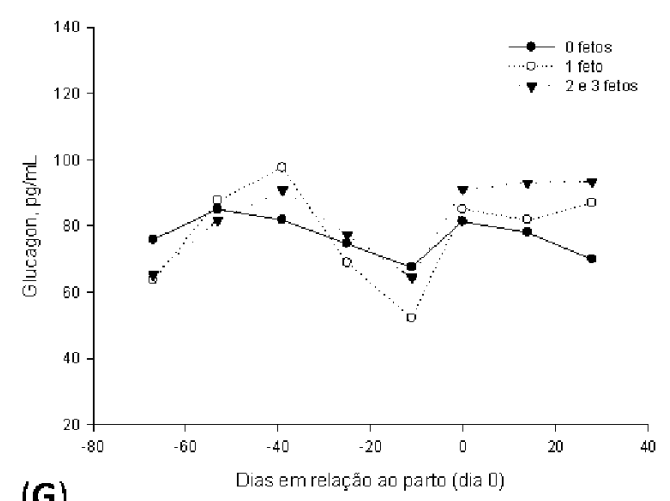

(G)

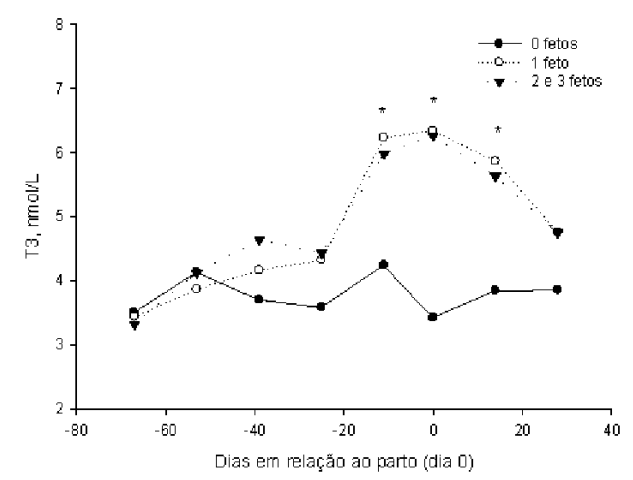

(B)

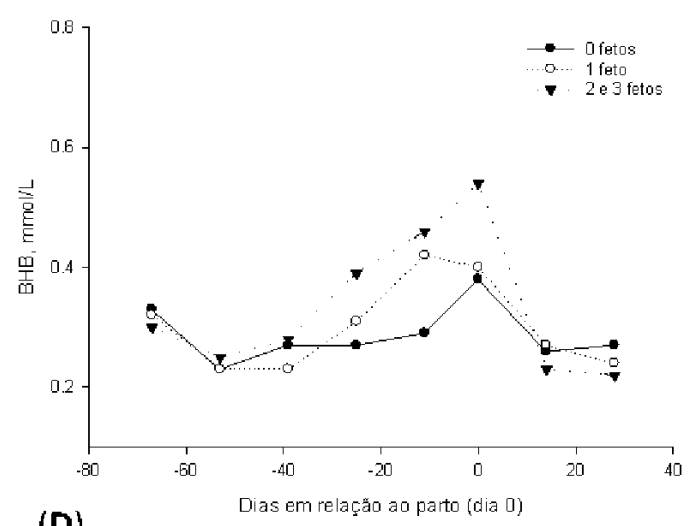

(D)

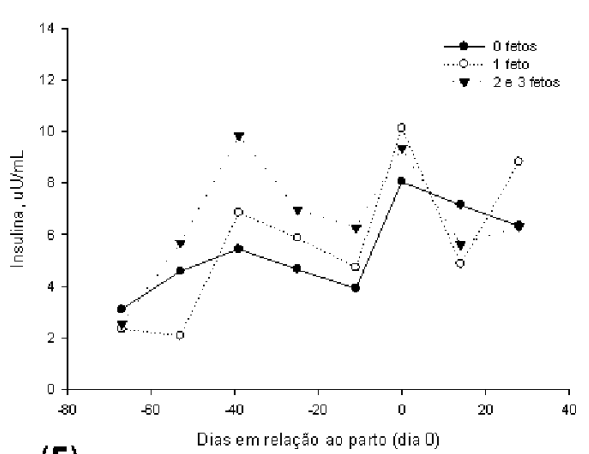

(F)

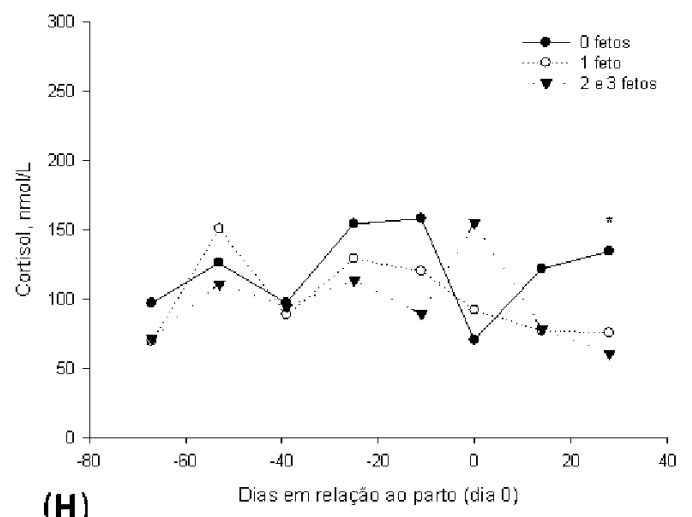

(H)

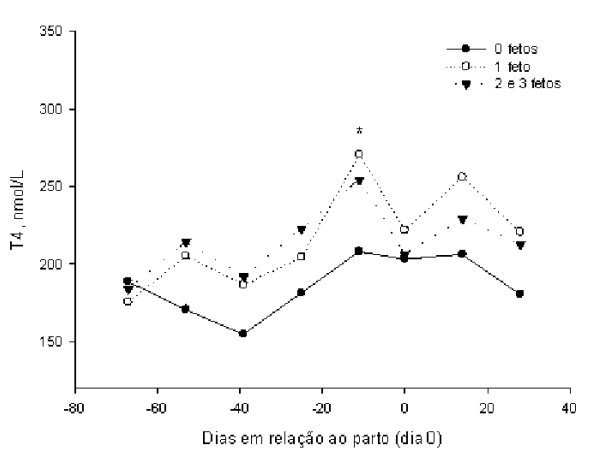

Fig.1. (A) Concentração plasmática de glicose, (B) betahidroxibutirato, (C) ácidos graxos não esterificados (NEFA), e (D) concentrações séricas de insulina, (E) glucacon, (F) cortisol, (G) T3 e (H) T4 de ovelhas da raça Santa Inês vazias (0 fetos), com gestação simples ( 1 feto) ou gestação gemelar ( 2 ou 3 fetos) durante a gestação, parto e puerpério. Dias de gestação das ovelhas prenhez de 1 ou 2 e 3 fetos: M1 = 66; M2 = 52; M3 = 38; M4 = 24; M5 = 10 dias anteriores ao parto; M6 = parto; M7 e M8 aos 14 e 28 dias pós-parto. Piracicaba. São Paulo, 2010. * Diferença entre tratamentos $(\mathrm{P}<0,05)$. 
Durante a gestação, os valores de glicemia permaneceram dentro dos limites de normalidade para espécie (2,8-4,5mmol/L) (Kaneko et al. 2008). Apenas no momento do parto estas concentrações se elevaram para as fêmeas gestantes tanto de feto único como de múltiplos, permanecendo os valores dentro da normalidade para fêmeas não gestantes. Santos et al. (2006) também observaram hiperglicemia apenas no momento do parto. 0 aumento nas concentrações de glicose observado no parto pode sugerir aumento das concentrações de glucagon e glicocorticoides que promovem a depleção dos estoques de glicogênio hepático (Grummer 1995) e/ou pode ser consequência da liberação de glicocorticóides como sinalizador do parto em ovinos (González 2000) corroborando com o encontrado no presente estudo, em que foi detectada relação baixa e moderada da glicemia com glucagon e com cortisol $(\mathrm{P}=0,001 ; \mathrm{r}=0,249$ e $\mathrm{r}=0,338$, respectivamente).

De acordo com Reece (2007), as concentrações de cortisol ficam aumentadas no final da gestação, pois funcionam como sinalizadores do momento do parto. Situações de estresse também podem provocar elevação nos teores deste glicocorticoide (Ford et al. 1990). Embora as ovelhas gestantes, tivessem apresentado hiperglicemia no momento do parto, não foi detectado aumento na concentração do cortisol no referido momento provavelmente pela coleta ter sido feita em momento posterior ao aumento do cortisol para desencadear o pico glicêmico já que foi considerado momento do parto até seis horas deste. Khan \& Ludri (2002) relatam, em cabras gestantes, que a concentração plasmática do cortisol do dia 20 ao dia 2 pré-parto diminuiu e 1 dia antes do parto elevou-se abruptamente chegando ao pico no momento do parto. A diferença detectada para o hormônio cortisol ocorreu somente 28 dias pós-parto. Este hormônio exerce papel importante no peri-parto devido a seu potente efeito gliconeogênico, entretanto, suas concentrações tendem a reduzir progressivamente no pós-parto em relação às últimas semanas de gestação (Campos et al. 2009). Como as fêmeas não manifestaram BEN intenso, a energia requerida para desenvolvimento dos fetos e produção de leite foi proveniente principalmente da alimentação, com pequena participação das reservas corporais.

A insulina é um importante hormônio do metabolismo energético e age aumentando a captação de glicose, estimulando seu armazenamento na forma de glicogênio e sua utilização como substrato para a lipogênese. Aumenta o fluxo na via glicolítica, inibe a glicólise e a gliconeogênese, reduzindo assim a produção hepática de glicose e inibindo a formação de corpos cetônicos (Berne e Levi 2004). As concentrações de insulina diminuem no período final de gestação e início da lactação em vacas leiteiras, com picos agudos no momento do parto (Kunz et al. 1985). Não foram encontradas diferenças quanto aos teores de insulina e glucagon entre os animais gestantes como também, entre gestantes e não gestantes provavelmente devido às condições de higidez e leve BEN que as ovelhas gestantes se encontravam.

Como não foram encontradas diferenças nas concentrações dos HT e AGNE entre as ovelhas com gestação única e as com gestação gemelar, conclui-se que devido à adequada quantidade energética da dieta, as ovelhas não entraram em intenso BEN e, portanto, nestas condições não há diferença entre os marcadores bioquímicos e hormonais aqui utilizados para ovelhas gestantes de um ou de dois e/ou três fetos.

\section{CONCLUSÕES}

A dieta oferecida neste estudo de 1,77 Mcal $/ \mathrm{kg} / \mathrm{MS}$ foi eficiente em evitar intenso balanço energético negativo no terço final da gestação. Ovelhas sadias e gestantes com leve BEN, independentemente do número de fetos, apresentaram maior atividade metabólica e mobilizaram mais reservas corporais que animais não gestantes no período periparto.

Nos casos de BEN não intenso, não há diferença entre as concentrações deAGNE, BHB, glicose, T3, T4, cortisol, insulina e glucagon em ovelhas hígidas e gestantes, independente do número de fetos.

Os resultados encontrados podem ser utilizados como valores de normalidade para ovelhas gestantes e ovelhas sadias.

Agradecimentos.- À Fundação de Amparo a Pesquisa do Estado de São Paulo (FAPESP), pela concessão de auxílio à pesquisa (no 2007/08729-9).

\section{REFERÊNCIAS}

Berne R.M. \& Levy M.N. 2004. Fisiologia. Elsevier, Rio de Janeiro. 1082p.

Caldeira R.M., Belo A.T., Santos C.C., Vazques M.I. \& Portugal A.V. 2007. The effect of body condition score on blood metabolites and hormonal profiles in ewes. Small Rum. Res. 68:233-241.

Campos R., Lacerda L.A., Terra S.R. \& González F.H.D. 2008. Parâmetros hematológicos e níveis de cortisol plasmático em vacas leiteiras de alta produção no sul do Brasil. Braz. J. Vet. Res. Anim. Sci. 45:354-361.

Campos R., Hérnandez E.A., Giraldo L. \& González F. 2009. Cortisol e sua relação com a regulação endócrina no período de transição em vacas leiteiras sob condições do trópico colombiano. Ciencia Animal Brasileira. Anais do VIII Congresso Brasileiro de Buiatria, Belo Horizonte, MG, p.790-794.

Celi P., Di Trana A. \& Claps S. 2008. Effects of perinatal nutrition on lactational performance, metabolic and hormonal profiles of dairy goats and respective kids. Small Rumin. Res. 79:129-136.

Contreras P.A., Phill M., Möller I., Wittwer F. \& Tadich N. 1990. Concentraciones sanguíneas de glucose, cholesterol, cuerpos cetónicos y actividad de aspartato aminotransferasa en ovejas con gestación única y gemelar en pastoreo rotacional intensivo. Arch. Méd. Vet. 22(1):65-69.

Emery R.S., Liesman J.S. \& Herdt T.H. 1992. Metabolism of long chain fatty acids by ruminant liver. J. Nutr. 122:832-837.

Ford E.J.H., Evans J. \& Robinson J. 1990. Cortisol in pregnancy toxemia of sheep. Brit. Vet. J. 146:539-542.

Fthenakis G.C., Arsenos G., Brozos C., Fragkou I.A., Giadinis N.D., Giannenas I., Mavrogianni V.S., Papadopoulos E. \& Valasi I. 2012. Health management of ewes during pregnancy. Anim. Reprod. Sci. 130:198-212

González F.H.D. 2000. Uso do perfil metabólico para determinar o status nutricional em gado de corte, p.63-74. In: González F.H.D., Barcellos J.O., Ospina H. \& Ribeiro L.A.O. (Eds), Perfil Metabólico em Ruminantes: seu uso em nutrição e doenças nutricionais. Gráfica da Universidade Federal do Rio Grande do Sul, Porto Alegre, RS. 108p.

Grummer R.R. 1995. Impact of changes in organic nutrient metabolism on feeding the transition dairy cow. J. Anim. Sci. 73:2820-2833.

Kaneko J.J., Harvey J.W. \& Bruss M.L. 2008. Clinical Biochemistry of Domestic Animals. $6^{\text {th }}$ ed. Academic Press, San Diego. 916p. 
Khan J.R. \& Ludri R.S. 2002. Hormone profile of crossbreed goats during the periparturient period. Trop. Anim. Health Prod. 34(2):151-162.

Kunz P.L., Blum J.W., Hart I.C.H. \& Landis J. 1985. Effects of different energy intakes before and after calving on food intake, performance and blood hormones and metabolites in dairy cows. Anim. Prod. Sci. 40:219-231.

Lima A. 2005. Avaliação do perfil hormonal de garrotes submetidos à carência energética prolongada. Dissertação de Mestrado, Universidade de São Paulo, São Paulo, SP. 139p.

Ortolani E.L. 1994. Doenças carenciais e metabólicas em caprinos: urolitíase e toxemia da prenhez. Anais do Encontro Nacional para o Desenvolvimento da Espécie Caprina, Unesp, Jaboticabal. 197p.

Payne J.M. \& Payne S. 1987. The Metabolic Profile. Oxford University Press, Oxford. 179p.

Reece W.0. 2007. Dukes: Fisiologia dos Animais Domésticos. 12ª ed. Guanabara Koogan, Rio de Janeiro. 926p.

Radostitis O.M. 2007. Veterinary Medicine. $10^{\text {th }}$ ed. W.B. Saunders, Edinburgh. 2156p.

Rook J.S. 2000. Pregnancy toxemia of ewes, does and beef cows. Vet. Clin. North Am., Food. Anim. Pract. 16(2):203-317.

Russel A.J.F. 1984. Body condition scoring of sheep. In Practice 6:91-93.
Santos J.R.S., Souza B.B., Souza W.H., Cezar M.F. \& Tavares G.P. 2006. Respostas fisiológicas e gradientes térmicos de ovinos das raças Santa Inês, Morada Nova e de seus cruzamentos com a raça Dorper às condições do semi árido nordestino. Ciênc. Agrotec. 30(5):995-1001.

Santos R.A., Campos A.G.S.S., Afonso J.A.B., Soares P.C. \& Mendonça C.L. 2012. Efeito da administração de propileno glicol e cobalto associado a vitamina $B_{12}$ sobre o perfil metabólico e a atividade enzimática de ovelhas da raça Santa Inês no periparto. Pesq. Vet. Bras. 32(Supl.1): 60-66.

Susin I. 1996. Exigências nutricionais de ovinos e estratégias de alimentação, p.119-141. In: Silva Sobrinho A.G., Batista A.M.V. \& Siqueira E.R. (Eds), Nutrição de Ovinos. Funep, Jaboticabal.

Todini L. 2007. Thyroid hormones in small ruminants: effects of endogenous, environmental and nutritional factors. Animal 1(7):997-1008.

Todini L., Malfatti A., Valbonesi A., Trabalza-marinucci M. \& Debenedetti A. 2007. Plasma total T3 and T4 concentrations in goats at different physiological stages, as affected by energy intake. Small Rumin. Res. 68:285290.

Yildiz A., Balikci E. \& Gurdogan F. 2005. Changes in some serum hormonal profiles during pregnancy in single and twin foetus bearing Akkaraman sheep. Medycyna Weterynaryjna 61:1138-1141. 